TIFR/TH/99-54

CERN-TH/99-321

October 1999

hep-th/9910194

\title{
D1/D5 System with B-field, Noncommutative Geometry and the CFT of the Higgs Branch
}

\author{
Avinash Dhar ${ }^{a}$, Gautam Mandal $^{a, c}$, Spenta R. Wadia ${ }^{a \dagger}$ \\ and K.P. Yogendran ${ }^{a}$ \\ ${ }^{a}$ Department of Theoretical Physics, Tata Institute of Fundamental Research, \\ Homi Bhabha Road, Mumbai 400 005, INDIA. \\ ${ }^{c}$ CERN Theory Division, CH-1211, Geneva 23, Switzerland.
}

\begin{abstract}
The D1/D5 system is considered in the presence of the NS B field. An explicit supergravity solution in the asymptotically flat and near horizon limits is presented. Explicit mass formulae are presented in both cases. This solution has no D3 source branes and represents a true bound state of the D1/D5 system. We study the motion of a separated D1-brane in the background geometry described above and reproduce the Liouville potential that binds the D1 brane. A gauge theory analysis is also presented in the presence of FayetIliopoulos (FI) parameters which can be identified with the self-dual part of the NS B field. In the case of a single D5-brane and an arbitrary number of D1 branes we can demonstrate the existence of a bound state in the Higgs branch. We also point out the connection of the SCFT on the resolved $\operatorname{Sym}_{Q_{1} Q_{5}}\left(\tilde{T}^{4}\right)$ with recent developments in non-commutative YangMills theory.
\end{abstract}

e-mail: adhar,mandal,wadia,kpy@theory.tifr.res.in

$\dagger$ Jawaharlal Nehru Centre for Advanced Scientific Research. Bangalore 560012, INDIA. 


\section{Introduction}

The D1/D5 system of type IIB string theory compactified on $S^{1} \times T^{4}$ (or type IIA on $S^{1} \times K^{3}$ ) has been the subject of many investigations since it was introduced by Strominger and Vafa [1] in their work on the microscopic origin of the entropy of the corresponding black hole solution in $(4+1)$-dimensions. This system is amenable to a rather precise analysis because its dynamics in the limit $\alpha^{\prime} \rightarrow 0$ is described by a non-abelian gauge theory. This fact underlies the hope that a correctly understood and calculable theory of Hawking radiation can emerge for the near extremal 5-dim. black hole. This hope is realized for the emissivity of the massless modes upto an over all numerical constant which can be fixed by assuming the AdS/CFT duality [2].

It turns out that the infra-red dynamics of D1/D5 system is described by a 2-dimensional superconformal sigma model whose target space is the moduli space of Yang-Mills instantons on $T^{4}$ or $K^{3}$. In this paper we will will restrict ourselves to $T^{4}$. This SCFT has a singularity corresponding to the vanishing size of instantons which corresponds to the origin of the Higgs branch. The singularity is resolved by turning on any one of the four exactly marginal operators which correspond to the blow-up modes. In the SCFT with target space $\operatorname{Sym}_{Q_{1} Q_{5}}\left(\tilde{T}^{4}\right)$ 円, these 4 operators arise from the twisted sector corresponding to a single nontrivial cycle of length 2 and are denoted by $\tau_{0}$ and $\tau_{1}$ in [3]. Using a global symmetry of the SCFT these were identified in [3] to be dual to the four supergravity fields: $B^{(+)}$(self-dual part of the NS 2-form $B$ ) and $a C^{(0)}+b C^{(4)}$ (a linear combination of the Ramond-Ramond forms $C^{(0)}$ and $C^{(4)}$ ). Seiberg and Witten [4 have shown that the singularity of the Higgs branch corresponds to the decay of the marginally stable bound system of D1/D5 branes to sub-systems of D1/D5 branes. Turning on any 4 marginal operators evades the singularity of the Higgs branch, because the marginally stable bound state is now expected to become a true bound state.

This fact can be argued in various ways and if we assume the AdS/CFT conjecture it certainly implies the existence of a black hole solution in the bulk where the moduli fields corresponding to the blowing up modes are non-zero. Such a black hole will be stable against fragmentation into constituents. In this paper we present such a solution for which $B \neq 0$ (in particular, $\left.B^{(+)}\right|_{\text {brane }} \neq 0$ ). A preliminary version was already reported in [5]. We present

\footnotetext{
${ }^{1} \mathrm{Sym}_{k} M$ denotes the symmetric product $M^{k} / S(k)$ where $S(k)$ is the permutation group of $k$ elements. The tilde on $T^{4}$ is a reminder that the 4 -torus is not necessarily identical to the original $T^{4}$ [2].
} 
a detailed analysis of the charges, both at infinity and at the horizon, to ensure that the system is indeed D1/D5 and there are no source D3-branes. Given this new solution we repeat the analysis in [4] and study the motion of a test D1 brane in the background of this solution. We demonstrate the Liouville potential that binds the D1 brane to a large number of D1/D5 branes. The coefficient of the Liouville potential is proportional to $b^{2}$, where $b$ is the self-dual part of the NS B field at the horizon.

The discussion of the bound state in the context of the microscopic theory can be done efficiently by approximating the instanton sector of the 5 -brane gauge theory by the $\mathcal{N}=4$, $U\left(Q_{1}\right) \times U\left(Q_{5}\right)$ gauge theory in 2 dims [6, 7]. We analyse the D-flatness (ADHM) equations when the Fayet-Iliopoulos (FI) parameters are non-zero, and indicate that the $(1,5)$ and $(5,1)$ strings condense on the hyper-Kahler manifold $T^{*} C P^{N-1}$, where $N=Q_{1} Q_{5}$. We then discuss the transverse fluctuations of the brane system (case $Q_{5}=1$ ) and show that these do not have flat directions transverse to the brane system. If the FI parameters are non-zero. This demonstrates a normalizable ground state of the Higgs branch.

As this work was getting completed we became aware of the recent developments in noncommutative geometry and string theory: [8, 9, 10, 11, 17, 12, 14, 13, 16, 18, 19, 15. The presence of the $B$ in the world volume of a collection of D-branes changes the boundary conditions of the open strings that mediate the interactions of the branes. The effect of this is that the Yang-Mills (YM) theory in 6-dimensions in $T^{4} \times S^{1} \times R^{1}$ that describes the collective modes of the D1/D5 system is now replaced by a noncommutative YM theory (NCYM), with the noncommutativity parameter determined by $B$, as explained in [9]. The moduli space to consider should now be that of the self-dual gauge fields in NCYM on the four-torus: $\hat{F}^{(-)}=0$, where $\hat{F}$ denotes the NCYM field strength and the superscript ${ }^{(-)}$ denotes anti-self-dual part, taken with respect to an appropriate (open string) metric. These conditions once again preserve 8 SUSY's and can be considered as a deformation of the original self dual equations.

In case of the YM theory in $R^{4}$, Nekrasov and Schwarz [20] have shown, by an analysis of the ADHM equations, that the moduli space of $\hat{F}^{(-)}=0$ is equivalent to a resolution of the moduli space of the ordinary $F^{(-)}=0$ by the $B_{i j}^{(+)}$(Fayet-Iliopoulos) parameters. For the case of the torus $T^{4}$ or $K^{3}$ we are unaware of a similar precise statement but an educated guess is the following: The moduli space of $\hat{F}_{i j}^{(-)}=0$ on the torus is a resolution of the symmetric product: $\operatorname{Sym}_{Q_{1} Q_{5}}\left(\tilde{T}^{4}\right)$. In applications, for instance, when one constructs the 
orbifold CFT on the symmetric product one implicitly assumes that a certain flux persists in the squashed 2 cycles. This flux corresponds to turning on the operator $\tau_{0}$ in [3]. We conjecture that the SCFT obtained by further adding the 3 operators $\tau_{1}$, with arbitrary values of the corresponding moduli, is the SCFT where target space is the moduli space of $F_{i j}^{(-)}=0$.

The paper is organized as follows: We first construct in Section 2, the supergravity solution that corresponds to the D1/D5 system in the presence of non-zero $B$ and the Liouville potential felt by a probe D1 brane. In Section 3, we discuss the $\mathcal{N}=4$, $U\left(Q_{1}\right) \times U\left(Q_{5}\right)$ gauge theory in 2 dimensions when the Fayet-Iliopoulos (FI) parameters are non-zero and discuss the transverse fluctuations of the brane system (case $Q_{5}=1$ ). We conclude with a proposal for the sigma model on the moduli space of NCYM instantons.

\section{D1/D5 System with B-field}

In this section we will construct a solution [5] to type IIB supergravity compactified on $T^{4}$ which represents the D1/D5 system with a NS B-field in the internal $T^{4}$ directions. We will obtain this solution from brane systems without a B-field by a series of T-dualities and use it to study various properties of this system discussed in the Introduction.

We will denote the $T^{4}$ directions as $x^{6,7,8,9}$. We begin with a IIB supergravity solution corresponding to two sets of orthogonal D3-branes extending along $x^{5,6,7}$ and $x^{5,8,9}$ respectively. The common direction, $x^{5}$, is assumed non-compact for the moment. The first set of D3-branes, which wraps the $T^{4}$ in $x^{6}$ and $x^{7}$ directions, is "smeared" (see, e.g. 22]) over the remaining $T^{4}$ directions, $x^{8}$ and $x^{9}$. The other set of D3-branes, which wraps $x^{8}$ and $x^{9}$ directions, is smeared over $x^{6}$ and $x^{7}$. This solution [23] is given by the following metric, dilaton and self-dual 5-form RR field strength:

$$
\begin{aligned}
d s^{2}= & \left(f_{1} f_{5}\right)^{-1 / 2}\left(-d t^{2}+\left(d x^{5}\right)^{2}\right)+\left(f_{1} f_{5}\right)^{1 / 2}\left(d r^{2}+r^{2} d \Omega_{3}^{2}\right) \\
& \left.+\left(\frac{f_{1}}{f_{5}}\right)^{1 / 2}\left(d x^{6}\right)^{2}+\left(d x^{7}\right)^{2}\right)+\left(\frac{f_{5}}{f_{1}}\right)^{1 / 2}\left(\left(d x^{8}\right)^{2}+\left(d x^{9}\right)^{2}\right) \\
e^{2 \phi}= & 1, \\
F^{(5)}= & K^{(3)} \wedge d x^{6} \wedge d x^{7}+\tilde{K}^{(3)} \wedge d x^{8} \wedge d x^{9}
\end{aligned}
$$


where

$$
\begin{aligned}
& K^{(3)}=\left(-d f_{5}^{-1} \wedge d t \wedge d x^{5}+\alpha^{\prime} \mu_{1} \epsilon_{3}\right) \\
& \tilde{K}^{(3)}=\left(-d f_{1}^{-1} \wedge d t \wedge d x^{5}+\alpha^{\prime} \mu_{5} \epsilon_{3}\right) .
\end{aligned}
$$

Here $\epsilon_{3}$ is the volume form of the 3 -sphere and the harmonic functions $f_{1}$ and $f_{5}$ are given by

$$
f_{1,5}=1+\frac{\mu_{1,5}}{2}\left(\frac{\alpha^{\prime}}{r^{2}}\right),
$$

where $\mu_{1,5}$ are dimensionless numbers related to the numbers of D3-branes in the two sets. We have used here a notation which is appropriate to the D1/D5 solution with a B-field that we are ultimately interested in. For the sake of completeness, we also give here the type IIB low-energy action to which the above is a solution:

$$
\begin{aligned}
S_{I I B}= & \frac{1}{2 \kappa^{2}} \int d^{10} x \sqrt{-G}\left\{e^{-2 \phi}\left(R+4(\nabla \phi)^{2}-\frac{1}{2.3 !}\left(H^{(3)}\right)^{2}\right)-\frac{1}{2.3 !}\left(F^{(3)}\right)^{2}\right. \\
& \left.-\frac{1}{4.5 !}\left(F^{(5)}\right)^{2}\right\}+\frac{1}{4 \kappa^{2}} \int C^{(4)} \wedge F^{(3)} \wedge H^{(3)}
\end{aligned}
$$

where $\left(H^{(3)}\right)^{2}=H_{\mathrm{MNP}}^{(3)} H^{(3) \mathrm{MNP}},\left(F^{(n)}\right)^{2}=F_{M_{1} \cdots M_{n}}^{(n)} F^{(n) M_{1} \cdots M_{n}}$ and, using the standard form notation,

$$
H^{(3)}=d B_{N S}^{(2)}, \quad F^{(3)}=d C^{(2)}, \quad F^{(5)}=d C^{(4)}-\frac{1}{2} C^{(2)} \wedge H^{(3)}+\frac{1}{2} B^{(2)} \wedge F^{(3)} .
$$

The self-duality constraint, $* F^{(5)}=F^{(5)}$, is imposed at the level of the equations of motion. Also, $\kappa^{2}=8 \pi G_{10}$, where $G_{10}=8 \pi^{6} g^{2} \alpha^{4}$ is the 10-dimensional Newton's constant (in the convention that the dilaton, $\phi$, vanishes asymptotically).

The desired D1/D5 solution is obtained from the above solution by the following series of T-duality transformations: A rotation in the $x^{6}-x^{8}$ plane by an angle $\varphi$, followed by a T-duality in the (new) direction $x^{8}$, followed by a rotation in the $x^{7}-x^{9}$ plane by an angle $\psi$, which is finally followed by a T-duality in the (new) direction $x^{9}$. We have used the T-duality rules for RR field strength in the form given in [25], adapted to our conventions (self-dual 5-form RR field strength). We present below the solution obtained in this way:

$$
\begin{aligned}
d s^{2}= & \left(f_{1} f_{5}\right)^{-1 / 2}\left(-d t^{2}+\left(d x^{5}\right)^{2}\right)+\left(f_{1} f_{5}\right)^{1 / 2}\left(d r^{2}+r^{2} d \Omega_{3}^{2}\right) \\
& +\left(f_{1} f_{5}\right)^{1 / 2}\left\{Z_{\varphi}^{-1}\left(\left(d x^{6}\right)^{2}+\left(d x^{8}\right)^{2}\right)+Z_{\psi}^{-1}\left(\left(d x^{7}\right)^{2}+\left(d x^{9}\right)^{2}\right)\right\},
\end{aligned}
$$




$$
\begin{aligned}
e^{2 \phi}= & f_{1} f_{5} / Z_{\varphi} Z_{\psi} \\
B_{N S}^{(2)}= & \left(Z_{\varphi}^{-1} \sin \varphi \cos \varphi\left(f_{1}-f_{5}\right)+b_{68}\right) d x^{6} \wedge d x^{8} \\
& +\left(Z_{\psi}^{-1} \sin \psi \cos \psi\left(f_{1}-f_{5}\right)+b_{79}\right) d x^{7} \wedge d x^{9} \\
F^{(3)}= & \cos \varphi \cos \psi \tilde{K}^{(3)}+\sin \varphi \sin \psi K^{(3)} \\
F^{(5)}= & Z_{\varphi}^{-1}\left(-f_{5} \cos \varphi \sin \psi K^{(3)}+f_{1} \cos \psi \sin \varphi \tilde{K}^{(3)}\right) \wedge d x^{6} \wedge d x^{8} \\
& +Z_{\psi}^{-1}\left(-f_{5} \cos \psi \sin \varphi K^{(3)}+f_{1} \cos \varphi \sin \psi \tilde{K}^{(3)}\right) \wedge d x^{7} \wedge d x^{9} \\
Z_{\varphi, \psi}= & 1+\frac{\mu_{\varphi, \psi}}{2}\left(\frac{\alpha^{\prime}}{r^{2}}\right), \quad \mu_{\varphi}=\mu_{1} \sin ^{2} \varphi+\mu_{5} \cos ^{2} \varphi, \quad \mu_{\psi}=\mu_{1} \sin ^{2} \psi+\mu_{5} \cos ^{2} \psi(2.9
\end{aligned}
$$

Here $b_{68}$ and $b_{79}$ are arbitrary constants which we have added at the end by a T-duality transformation that shifts the NS B-field by a constant. Note that for $\varphi=\psi=0$ and $b_{68}=b_{79}=0$, the above solution reduces to the well-known solution for D1/D5 system without B-field.

\subsection{Charges}

In this subsection we will discuss the various charge densities 2 associated with the RR fields. The electric charge densities are given by

$$
Q_{\mathrm{elec}}=\frac{1}{2 \kappa^{2}} \oint * F^{(n)}
$$

and the magnetic charge densities by

$$
Q_{\mathrm{mag}}=\frac{1}{2 \kappa^{2}} \oint F^{(n)}
$$

The integrals above are evaluated at asymptotic distances in the 4-dimensional noncompact space. Note that for arbitrary $\varphi$ and $\psi$ values, $F^{(3)}$ and $F^{(5)}$ have non-trivial $r$-dependence which is different from the asymptotic dependence $1 / r^{3}$. This means, in particular, that the numerical values of the charges computed in the asymptotically flat geometry are different from those computed in the near horizon AdS geometry. We shall give below expressions for charges in both cases. The charges in the near horizon geometry have been distinguished from those in the asymptotically flat geometry by a superscript ${ }^{(h)}$.

\footnotetext{
${ }^{2}$ These are densities rather than the total charges, as explained below (2.13).
} 
The RR field strength $F^{(3)}$ gives rise to D1-brane and D5-brane charges while $F^{(5)}$ gives rise to two types of D3-brane electric and magnetic charges. For our solution these charge densities are, in an obvious notation,

$$
\begin{aligned}
Q_{1} & =\beta\left(\mu_{1} \cos \varphi \cos \psi+\mu_{5} \sin \varphi \sin \psi\right), \\
Q_{5} & =\beta\left(\mu_{5} \cos \varphi \cos \psi+\mu_{1} \sin \varphi \sin \psi\right), \\
Q_{3} \equiv Q_{3}^{568} & =\beta\left(\mu_{5} \cos \varphi \sin \psi-\mu_{1} \cos \psi \sin \varphi\right), \\
Q_{3}^{\prime} \equiv Q_{3}^{579} & =\beta\left(\mu_{5} \cos \psi \sin \varphi-\mu_{1} \cos \varphi \sin \psi\right),
\end{aligned}
$$

where $\beta=\alpha^{\prime} \Omega_{3} /\left(2 \kappa^{2}\right)$ and $\Omega_{3}=2 \pi^{2}$ is the volume of a unit 3 -sphere. Here $Q_{1}$ is the charge 'density' of D1-branes obtained by dividing in addition by the asymptotic volume of the torus $T^{4}$ and $Q_{3}, Q_{3}^{\prime}$ are D3-brane charge densities obtained by dividing in addition by appropriate $T^{2}$ volumes.

In the near horizon geometry, the corresponding expressions for the charge densities are,

$$
\begin{aligned}
Q_{1}^{(h)} & =\beta \frac{\mu_{1} \mu_{5}}{\mu_{\varphi} \mu_{\psi}}\left(\mu_{5} \cos \varphi \cos \psi+\mu_{1} \sin \varphi \sin \psi\right), \\
Q_{5}^{(h)} & =Q_{5} \\
Q_{3}^{(h)} \equiv Q_{3}^{568(h)} & =\beta \frac{\mu_{1} \mu_{5}}{\mu_{\psi}} \sin (\psi-\varphi), \\
Q_{3}^{(h)^{\prime}} \equiv Q_{3}^{579(h)} & =\beta \frac{\mu_{1} \mu_{5}}{\mu_{\varphi}} \sin (\varphi-\psi) .
\end{aligned}
$$

As expected, only the D5-brane charge density is the same in the two cases.

\subsection{Source Branes and Mass}

We now require that our solution should correspond to only D1- and D5-brane sources. That is, we want the D3-brane charges to be induced purely by our nonzero B-field, and not by any source D3-branes.

There are two contributions to the D3-brane charge induced by the B-field. One comes from the bulk Chern-Simons term in (2.2),

$$
\frac{1}{4 \kappa^{2}} \int C^{(4)} \wedge F^{(3)} \wedge H
$$

\footnotetext{
${ }^{3}$ Note that the full space of supergravity solutions generated by the T-duality group $O(4,4)$ will have arbitrary number of source D1-,D3- and D5-branes. We will choose below parameters of our T-duality transformations in such a way that we have a 'pure D1/D5 system'.
} 
and the other from the coupling

$$
Q_{5} \int C^{(4)} \wedge B
$$

in the Chern-Simons part of the D5-brane would volume action,

$$
\rho_{(5)} \operatorname{Tr} \int \sum_{n} e^{2 \pi \alpha^{\prime} F+B} \wedge C^{(n)}
$$

Here $\rho_{(5)}$ is the charge density of a D5-brane and the trace is over the non-abelian gauge group $\mathcal{U}\left(n_{5}\right), n_{5}$ being the number of D5-branes.

The coupling in (2.18) contributes a $\delta$-function term (with support at the location of the D5-brane in the 4 noncompact dimensions) in the local D3-brane charge density, proportional to the value of the B-field at the horizon. On the other hand, the contribution of (2.19) to the D3-brane charge is proportional to the difference of the asymptotic and the horizon values of B. It can be shown that the sum of these two contributions is an induced D3-brane charge proportional to the asymptotic value of the B field, thus leading to (2.22) below.

Similarly to the above, there are also contributions to the total D1-brane charge which

are induced by the B-field. Apart from the bulk Chern-Simons term (2.19), there is also a contribution from the D5-brane

Chern-Simons action which is given by

$$
Q_{5} \int \frac{1}{2 !} B \wedge B \wedge C^{(2)}
$$

In this case also the two contributions add up to finally give induced D1-brane charge which depends only on the asymptotic value of $\mathrm{B}$, leading to (2.26) below.

Let us now discuss the two cases of the asymptotically flat and the near horizon geometry separately. In other words, we will consider first the case of the full supergravity solution (2.4) and then the case in which we take the near-horizon limit in the spirit of [26] and treat it as an independent solution of IIB supergravity in its own right.

(i) Asymptotically flat geometry

Here, the absence of any D3-brane sources is ensured by demanding that

$$
Q_{3}=B_{79}^{(\infty)} Q_{5}, \quad Q_{3}^{\prime}=B_{68}^{(\infty)} Q_{5}
$$


where

$$
B_{79}^{(\infty)}=b_{79}, \quad B_{68}^{(\infty)}=b_{68}
$$

are the asymptotic values of the two nonzero components of the B-field. Eqns. (2.22) are satisfied if we set

$$
\begin{aligned}
b_{68} & =\frac{\mu_{5} \sin \varphi \cos \psi-\mu_{1} \cos \varphi \sin \psi}{\mu_{5} \cos \varphi \cos \psi+\mu_{1} \sin \varphi \sin \psi} \\
b_{79} & =\frac{\mu_{5} \cos \varphi \sin \psi-\mu_{1} \sin \varphi \cos \psi}{\mu_{5} \cos \varphi \cos \psi+\mu_{1} \sin \varphi \sin \psi}
\end{aligned}
$$

Furthermore, if $Q_{1 s}$ denotes the D1-brane charge which arises from source D1-branes, then, using (2.2) and (2.21) it can be shown that

$$
Q_{1 s}=Q_{1}-B_{68}^{(\infty)} B_{79}^{(\infty)} Q_{5}
$$

From (2.10)-(2.13) and (2.22)-(2.26) we then get

$$
Q_{1 s}=\beta^{2} \frac{\mu_{1} \mu_{5}}{Q_{5}}
$$

$\underline{\text { Mass }}$

The supergravity solution that we have is $\frac{1}{4}$ BPS. The mass of this $\frac{1}{4}$ BPS solution [27], which coincides with its ADM mass, is given in terms of the appropriate charge densities by

$$
M^{2}=\left(Q_{1}+Q_{5}\right)^{2}+\left(Q_{3}-Q_{3}^{\prime}\right)^{2}
$$

Note that, as expected, the mass given by (2.28) equals the ADM mass of

the intersecting D3-brane solution from which the present solution was obtained by Tduality, i.e.

$$
M=\beta\left(\mu_{1}+\mu_{5}\right) .
$$

We wish to study $M$ as a function of the moduli, keeping $Q_{1 s}$ and $Q_{5}$ fixed. This last requirement, together with (2.24) and (2.25), determines $\mu_{1}, \mu_{5}, \varphi$ and $\psi$ as functions of the two moduli $b_{68}$ and $b_{79}$ :

$$
\begin{aligned}
\tan (\varphi \pm \psi) & =\frac{b_{68} \pm b_{79}}{1 \mp b_{68} b_{79} \mp Q_{1 s} / Q_{5}} \\
\beta \mu_{1} & =\frac{Q_{5}}{2}\left\{\left(1+b_{68} b_{79}+Q_{1 s} / Q_{5}\right) \sec (\varphi-\psi)\right.
\end{aligned}
$$




$$
\begin{array}{r}
\left.-\left(1-b_{68} b_{79}-Q_{1 s} / Q_{5}\right) \sec (\varphi+\psi)\right\}, \\
\beta \mu_{5}=\frac{Q_{5}}{2}\left\{\left(1+b b^{\prime}+Q_{1 s} / Q_{5}\right) \sec (\varphi-\psi)\right. \\
\left.+\left(1-b_{68} b_{79}-Q_{1 s} / Q_{5}\right) \sec (\varphi+\psi)\right\} .
\end{array}
$$

For arbitrary $b_{68}$ and $b_{79}, \mu_{1}$ and $\mu_{5}$ given by the above equations will not satisfy any quantization conditions, unlike, e.g., for $b_{68}=b_{79}=0$ for which $\mu_{5}=2 g_{s} n_{5}$ and $\mu_{1}=\frac{2 g_{s} n_{1}}{V_{T^{4}} /(2 \pi)^{4} \alpha^{\prime 2}}$, where $n_{1}$ and $n_{5}$ are integers. This should not unduly worry us since for the system under discussion only $Q_{1 s}$ and $Q_{5}$ have a direct physical interpretation in terms of brane sources, and it is only on these that appropriate quantization conditions must be imposed.

We now wish to extremize $(2.28)$ with respect to both $b_{68}$ and $b_{79}$. This requires

$$
b_{68}=-b_{79}= \pm \sqrt{Q_{1 s} / Q_{5}-1}
$$

and then the mass at the fixed point is given by

$$
M^{2}=4 Q_{1 s} Q_{5}
$$

For fixed asymptotic volume of $T^{4}$, Eqns. (2.33) can be seen to fix the $B$-moduli at the minimum in terms of the ratio of D1- and D5-brane source charges. For a different value for this ratio, the mass is minimized for appropriately different values of the $B$-moduli. Stated differently, for a fixed value of the $B$-moduli, unless the charge-ratio is given by (2.33), the system will be unstable and decay to a system which satisfies (2.33).

Hence, once the B-moduli are fixed at the values in (2.33), the system forms a true bound state of the constituent branes 1 .

(ii) Near horizon geometry

In this case, absence of D3-brane sources is ensured if we set

$$
Q_{3}^{(h)}=B_{79}^{(h)} Q_{5}, \quad Q_{3}^{(h)^{\prime}}=B_{68}^{(h)} Q_{5},
$$

\footnotetext{
${ }^{4}$ It is interesting to consider the case when the fragmentation happens keeping the ratio of $Q_{1 s} / Q_{5}$ fixed. Eqn. (2.34) would predict zero binding energy and fragmentation at no cost. However, such an eventuality will be prevented for mutually prime $Q_{1 s}, Q_{5}$. This is in accord with [28, 4, 29] which indicate singularities associated with points in the moduli space where $Q 1 s$ and $Q_{5}$ have common factors.
} 
where

$$
\begin{aligned}
B_{68}^{(h)} & =\frac{\mu_{1}-\mu_{5}}{\mu_{\varphi}} \sin \varphi \cos \varphi+b_{68}, \\
B_{79}^{(h)} & =\frac{\mu_{1}-\mu_{5}}{\mu_{\psi}} \sin \psi \cos \psi+b_{79},
\end{aligned}
$$

are the horizon values of the two nonzero components of the B-field. Eqns. (2.35) are satisfied for precisely the same values of $b_{68}$ and $b_{79}$ as in (2.24) and (2.25). Moreover, we see that in this case

$$
\frac{B_{68}^{(h)}}{\mu_{\psi}}=-\frac{B_{79}^{(h)}}{\mu_{\varphi}}
$$

which is the self-duality condition on the B-field in the near horizon geometry. We also note that the volume of $T^{4}$ at the horizon is given by

$$
V_{T^{4}}^{(h)}=\frac{\mu_{1} \mu_{5}}{\mu_{\varphi} \mu_{\psi}}=\frac{Q_{1}^{(h)}}{Q_{5}} .
$$

The D1-brane charge that arises from source D1-branes in this case is given by

$$
Q_{1 s}^{(h)}=Q_{1}^{(h)}-B_{68}^{(h)} B_{79}^{(h)} Q_{5}
$$

From (2.14)-(2.17) and (2.35) we get

$$
Q_{1 s}^{(h)}=Q_{1 s}
$$

where $Q_{1 s}$ is given by (2.27). Thus we see that not only do the parameters $b_{68}$ and $b_{79}$ have the same values here as in the asymptotically flat case, even the source D1-branes are identical, despite the total D1-brane charges being very different in the two cases.

$\underline{\text { Mass }}$

The $\frac{1}{4}$ BPS mass formula in terms of the various charge densities in this case is

$$
\left(\frac{M^{(h)}}{V_{T^{4}}^{(h)}}\right)^{2}=\left(\frac{Q_{1}^{(h)}}{V_{T^{4}}^{(h)}}+Q_{5}\right)^{2}+\left(\frac{Q_{3}^{(h)}}{\sqrt{g_{77} g_{99}}}-\frac{Q_{3}^{(h)^{\prime}}}{\sqrt{g_{66} g_{88}}}\right)^{2} .
$$

Using (2.35)-(2.41) it can be easily seen that

$$
\left(M^{(h)}\right)^{2}=V_{T^{4}}^{(h)}\left(4 Q_{1 s} Q_{5}\right)
$$


Apart from the extra factor of the $T^{4}$ volume in the near horizon geometry, this is exactly the same as (2.34). The extra volume factor correctly takes into account the difference in the 6dimensional Newton's constant between the asymptotically flat and near horizon geometries because of the difference in the $T^{4}$ volume in the two cases. We have already seen that the B-field is automatically self-dual in the near horizon geometry and that the volume of $T^{4}$ satisfies the condition given by (2.39) and (2.40). We now see that the mass of the bound state is already at the fixed point value. Thus the solution we have here provides an explicit demonstration of the attractor mechanism of [30].

\subsection{Motion of a separated D1-brane}

In this subsection we will discuss the fragmentation of the D1/D5 system in which a single D1-brane splits off. As mentioned above, the presence of a $B$-field leads to a bound state. Therefore, if we consider the motion of the single D1-brane in the background geometry of the remaining branes, we should find an attractive potential. This problem was addressed in [4] who argued that the potential is of the Liouville form in the near-horizon geometry. We will use our supergravity solution to find the attractive potential in the full geometry which will include the above result in the near-horizon limit.

Let us assume that the remaining D1/D5 system is given by the parameters $Q_{1}, Q_{5}$. The motion of the separated D1-brane can then be described by the following DBI action, coupled to the supergravity solution (2.4)-(2.9)

$$
S_{1}=-\frac{1}{2 \pi g \alpha^{\prime}} \int d^{2} \sigma\left(e^{-\phi} \sqrt{-\operatorname{det}\left(\hat{g}+2 \pi \alpha^{\prime} \hat{B}\right)}-C^{(2)}\right)
$$

where

$$
\hat{g}_{\alpha \beta}=g_{\mu \nu} \partial_{\alpha} x^{\mu} \partial_{\beta} x^{\nu}, \quad \hat{B}_{\alpha \beta}=B_{\mu \nu} \partial_{\alpha} x^{\mu} \partial_{\beta} x^{\nu}
$$

represent the pull-backs of the metric and the $B$-field onto the D1-brane world-sheet. We will consider a radially moving D1-brane, given by

$$
x^{0}=\sigma_{0} \equiv \tau, x^{5}=\sigma_{1} \equiv \sigma, r=r(\tau)
$$

It is easy to find $S_{1}$ explicitly for small $B$-moduli f, (small angles $\phi, \psi$ ):

$$
S_{1}=\frac{1}{g \alpha^{\prime}} \int d \tau\left(\phi \psi f_{5}^{-1}\right)+o\left(v^{2}\right)
$$

\footnotetext{
${ }^{5}$ For radial motion, $\hat{B}$ vanishes, but the effect of non-zero $B$ shows up in the presence of non-zero angles $\phi, \psi$ which leads to non-zero $C^{(2)}$.
} 
As we have argued above, the minimum-energy bound state requires the condition (2.33), which in turn means $\phi=-\psi$. For small $B$-moduli, therefore, the coefficient of $f_{5}^{-1}$ is $-\phi^{2}=-b^{2}$, where $b=b_{68}=-b_{79}$.

Thus the separated D1-brane feels an attractive potential

$$
V(r)=\frac{1}{g \alpha^{\prime}} b^{2}\left(1+\frac{\mu_{5} \alpha^{\prime}}{2 r^{2}}\right)^{-1}
$$

The near-horizon limit is given by the functional form $r^{2}$ which, in terms of the scalar field $\phi=\ln r$ on the throat, is of the Liouville form $e^{2 \phi}$.

It is remarkable that the separated D1-brane feels a static potential which is the hallmark of a non-BPS situation, whereas normally one expects a Dp-Dq system with $p-q$ $\bmod 4=0$ to be BPS. It appears even more remarkable if one recalls that the supergravity solution with the B-field, which shows the non-zero binding energy, is obtained simply by a series of T-duality transformations. Appearance of non-zero binding energy as a result of T-duality has been noted before (see, e.g., [24]). See also related remarks in [9].

\section{Gauge Theory Description of the D1-D5 Bound State}

In this section we discuss the microscopic theory of the $D 1-D 5$ system. The moduli space of instantons of the 5-brane gauge theory with gauge group $U\left(Q_{5}\right)$ and instanton number $Q_{1}$ is the resolved $\operatorname{Sym}_{Q_{1} Q_{5}}\left(\tilde{T}^{4}\right)$. [. In the limit of small instanton size one can approximate this description by directly constructing the gauge theory corresponding to the system of $Q_{1}$ D1-branes and $Q_{5}$ D5-branes [6], [0]. This description provides a good physical picture of the dynamics of the

brane system. The brane configuration is as follows. The D1-branes are wrapped on the circle $S^{1}$ along the direction $x_{5}$ and the D5-branes are wrapped on the 5-torus $S^{1} \times T^{4}$. The coordinates of $T^{4}$ are denoted by $x_{i}, i=6,7,8,9$. The radius $R$ of $S^{1}$ is chosen so that $R \gg \sqrt{\alpha^{\prime}}$ and $V_{4} \sim \alpha^{\prime 2}$.

The above configuration of branes interact via open strings that are attached to the branes. Denote these by $(1,1),(5,5),(1,5)$ and $(5,1)$. The low energy dynamics of this

\footnotetext{
${ }^{6}$ We have here dropped the subscript 's' on the source D1-brane charge (which corresponds now to the instanton number) and, furthermore, by abuse of notation, $Q_{5}$ and $Q_{1}$ are now representing the corresponding numbers of branes rather than appropriate charge densities as in the previous section.
} 
system is described by a $U\left(Q_{1}\right) \times U\left(Q_{5}\right), N=4$ gauge theory in the 2 dimensions common to the 2 branes i.e. $\left(t, x_{5}\right)$. We also allow for a non-zero $\theta$ angle corresponding to the relative $U(1)$ that acts on the hypermultiplets.

The field content is summarized as follows:

(i) $(1,1)$ Strings :

The fields corresponding to the massless excitations of the $(1,1)$ strings are obtained from the dimensional reduction of $U\left(Q_{1}\right), N=1 \mathrm{SYM}$ in 10-dim. The bosonic fields can be organized as vector and hyper multiplets of $N=2$ theory in 4-dim. in the adjoint representation. of $U\left(Q_{1}\right)$ :

$$
\begin{aligned}
& \text { vector multiplets }: A_{0}^{(1)}, A_{5}^{(1)}, Y_{m} \quad m=1,2,3,4 \\
& \text { hyper multiplets }: Y_{i}, \quad i=6,7,8,9 .
\end{aligned}
$$

In the above description we have neglected the winding modes of the $(1,1)$ strings on the torus $T^{4}$.

(ii) $(5,5)$ Strings :

The massless modes of the $(5,5)$ open strings are obtained in a similar way except that the gauge group is $U\left(Q_{5}\right)$ and we neglect the $K K$ modes corresponding to the torus $T^{4}$.

$$
\begin{aligned}
& \text { vector multiplets }: A_{0}^{(5)}, A_{5}^{(5)}, X_{m} \quad m=1,2,3,4 \\
& \text { hyper multiplets }: \quad X_{i}, \quad i=6,7,8,9 .
\end{aligned}
$$

\section{(iii) $(1,5)$ and $(5,1)$ Strings :}

The fields corresponding to the massless excitations of the $(1,5)$ and $(5,1)$ open strings can be organized as doublets of the $S U(2)_{R}$ symmetry of the gauge theory. $S U(2)_{R}$ is the diagonal subgroup of $S O(4)_{I} \simeq S U(2) \times S U(2)$ which acts on the coordinates $x_{i}=6,7,8,9$.

$$
\text { hyper multiplets : } \chi_{a^{\prime} a}=\left(\begin{array}{c}
\chi_{a^{\prime} a}^{1} \\
\chi_{a^{\prime} a}^{2}
\end{array}\right)=\left(\begin{array}{c}
A_{a^{\prime} a} \\
B_{a^{\prime} a}
\end{array}\right)
$$

$A_{a^{\prime} b}$ and $B_{a^{\prime} b}$ transform in the bi-fundamental representation of $U\left(Q_{1}\right) \times \overline{U\left(Q_{5}\right)}$. The $U(1) \times \overline{U(1)}$ subgroup is important. One combination leaves the hypermultiplet 
invariant. The other combination is active and $\left(A_{a^{\prime} a}, B_{a^{\prime} a}\right)$ have $U(1)$ charges $(+1,-1)$. $\chi$ is a chiral spinor of $S O(4)_{I}$ with convention $\Gamma_{6789} \chi=-\chi$.

In the above discussion, the fields $Y_{i}$ and $X_{i}$ along the torus directions are assumed to be compact. However it is not obvious how to compactify the range of $\chi$ so that the integration over this field in the path integral is finite.

\subsection{The Potential Terms}

The potential energy density of the vector and hyper multiplets is a sum of 4 positive terms,

$$
\begin{aligned}
V= & V_{1}+V_{2}+V_{3}+V_{4} \\
V_{1}= & -\frac{1}{4 g_{1}^{2}} \sum_{m, n} \operatorname{tr}_{U\left(Q_{1}\right)}\left[Y_{m}, Y_{n}\right]^{2}-\frac{1}{4 g_{5}^{2}} \sum_{m, n} \operatorname{tr}_{U\left(Q_{5}\right)}\left[X_{m}, X_{n}\right]^{2} \\
V_{2}= & -\frac{1}{2 g_{1}^{2}} \sum_{i, m} \operatorname{tr}_{U\left(Q_{1}\right)}\left[Y_{i}, Y_{m}\right]^{2}-\frac{1}{2 g_{5}^{2}} \sum_{i, m}\left[X_{i}, X_{m}\right]^{2} \\
V_{3}= & \frac{1}{4} \sum_{m} \operatorname{tr}_{U\left(Q_{1}\right)}\left(\chi X_{m}-Y_{m} \chi\right)\left(X_{m} \chi^{\dagger}-\chi^{\dagger} Y_{m}\right)^{2} \\
V_{4}= & \frac{1}{4} \operatorname{tr}_{U\left(Q_{1}\right)}\left(\chi i \Gamma_{i j}^{T} \chi^{+}+i\left[Y_{i}, Y_{j}\right]^{+}-\zeta_{i j}^{+} \frac{1}{Q_{1}}\right)^{2} \\
& +\frac{1}{4} \operatorname{tr}_{U\left(Q_{5}\right)}\left(\chi^{+} i \Gamma_{i j} \chi+i\left[X_{i}, X_{j}\right]^{+}-\zeta_{i j}^{+} \frac{1}{Q_{5}}\right)^{2}
\end{aligned}
$$

The potential energy $V_{4}$ comes from a combination of $F$ and $D$ terms of the higher dim. gauge theory. $\Gamma_{i j}=\frac{i}{2}\left[\Gamma_{i}, \Gamma_{j}\right]$ are spinor rotation matrices. The notation $a_{i j}^{+}$denotes the self-dual part of the anti-symmetric tensor $a_{i j}$.

In $V_{4}$ we have included the Fayet-Iliopoulos (FI) terms $\zeta_{i j}^{+}$, which form a triplet under $S U(2)_{R}$. Their inclusion is consistent with $N=4$ SUSY. The FI terms can be identified with the self dual part of $B_{i j}$, the anti-symmetry tensor of the NS sector of the closed string theory. This identification at this stage rests on the fact that (i) $\zeta_{i j}^{+}$and $B_{i j}^{+}$have identical transformation properties under $S U(4)_{I}$ and (ii) at the origin of the Higgs branch where $\chi=X=Y=0, V_{4} \sim \zeta_{i j}^{+} \zeta_{i j}^{+}$. This signals a tachyonic mode from the view point of string perturbation theory. The tachyon mass is easily computed and this implies the relation $\zeta_{i j}^{+} \zeta_{i j}^{+} \sim B_{i j}^{+} B_{i j}^{+}$. 


\subsection{D-Flatness Equations and the Moduli Space}

The supersymmetric ground state (semi-classical) is characterized by the 2-sets of D-flatness equations which are obtained by setting $V_{4}=0$. They are best written in terms of the $S U(2)_{R}$ doublet fields $N_{a^{\prime} b^{\prime}}^{(1)}$ and $N_{a b}^{(5)}$ :

$$
\begin{aligned}
& N^{(1)}=\left(\begin{array}{l}
N_{1}^{(1)} \\
N_{2}^{(1)}
\end{array}\right)=\left(\begin{array}{c}
Y_{9}+i Y_{8} \\
Y_{7}+i Y_{6}
\end{array}\right) \\
& N^{(5)}=\left(\begin{array}{l}
N_{1}^{(5)} \\
N_{2}^{(5)}
\end{array}\right)=\left(\begin{array}{l}
X_{9}+i X_{8} \\
X_{7}+i X_{9}
\end{array}\right)
\end{aligned}
$$

We also define $\zeta=\zeta_{69}^{+}$and $\zeta_{c}=\zeta_{67}^{+}+i \zeta_{68}^{+}$. With these definitions the 2 sets of D-flatness conditions become:

$$
\begin{gathered}
\left(A A^{+}-B^{+} B\right)_{a^{\prime} b^{\prime}}+\left[N_{1}^{(1)}, N_{1}^{(1) \dagger}\right]_{a^{\prime} b^{\prime}}-\left[N_{2}^{(1)}, N_{2}^{(1) \dagger}\right]_{a^{\prime} b^{\prime}}=\frac{\zeta}{Q_{1}} \delta_{a^{\prime} b^{\prime}} \\
(A B)_{a^{\prime} b^{\prime}}+\left[N_{1}^{(1)}, N_{2}^{(1) \dagger}\right]_{a^{\prime} b^{\prime}}=\frac{\zeta_{c}}{Q_{1}} \delta_{a^{\prime} b^{\prime}} \\
\left(A^{+} A-B B^{+}\right)_{a b}+\left[N_{1}^{(5)}, N_{1}^{(5) \dagger}\right]_{a b}-\left[N_{2}^{(5)}, N_{2}^{(5) \dagger}\right]_{a b}=\frac{\zeta}{Q_{5}} \delta_{a b} \\
\left(A^{+} B^{+}\right)_{a b}+\left[N_{1}^{(5)}, N_{2}^{(5) \dagger}\right]_{a b}=\frac{\zeta_{c}}{Q_{5}} \delta_{a b}
\end{gathered}
$$

The hypermultiplet moduli space is a solution of the above equations modulo the gauge group $U\left(Q_{1}\right) \times U\left(Q_{5}\right)$. A detailed discussion of the procedure was given in [7]. Here we summarize.

If we take the trace parts of Eqns. (3.8) we get the same set of 3 equations as the Dflatness equations for a $U(1)$ theory with $Q_{1} Q_{5}$ hypermultiplets, with $U(1)$ charge assignment $(+1,-1)$ for $\left(A_{a^{\prime} b}, B_{a^{\prime} b}^{T}\right)$. Thus,

$$
\begin{gathered}
\sum_{a^{\prime} b}\left(A_{a^{\prime} b} A_{a^{\prime} b}^{*}-B_{a^{\prime} b}^{T} B_{a^{\prime} b}^{T *}\right)=\zeta \\
\sum_{a^{\prime} b} A_{a^{\prime} b} B_{a^{\prime} b}^{T}=\zeta_{c}
\end{gathered}
$$

For a given point on the surface defined by Eqns. (3.9) the traceless parts of (3.8) lead to $3 Q_{1}^{2}+3 Q_{5}^{2}-6$ constraints on among $4 Q_{1}^{2}+4 Q_{5}^{2}-8$ degrees of freedom corresponding to the traceless parts of the adjoint hypermultiplets $N^{(1)}$ and $N^{(5)}$. Using $Q_{1}^{2}+Q_{5}^{2}-2$ gauge conditions corresponding to $S U\left(Q_{1}\right) \times S U\left(Q_{5}\right)$ we have $\left(3 Q_{1}^{2}+3 Q_{5}^{2}-6\right)+\left(Q_{1}^{2}+Q_{5}^{2}-2\right)=$ 
$4 Q_{1}^{2}+4 Q_{5}^{2}-8$ conditions for the $\left(4 Q_{1}^{2}+4 Q_{5}^{2}-8\right)$ degrees of freedom in the traceless parts of $N^{(1)}$ and $N^{(5)}$. The 8 degrees of freedom corresponding to $\operatorname{tr} X_{i}$ and $\operatorname{tr} Y_{i}, i=6,7,8,9$ correspond for the centre-of-mass of the D5 and D1 branes respectively.

The Eqns.(3.9) for $Q_{1} Q_{5}$ hypermultiplets have been recently discussed in the context of instantons in non-commutative gauge theory [20, 9]. They define $M=T^{*} C P^{Q_{1} Q_{5}-1}$, the cotangent bundle of the complex hyper-Kähler manifold $C P^{Q_{1} Q_{5}-1}$. The FI parameters $\zeta_{i j}^{+}$ can be identified with the moduli of the hyper-Kähler metrics on $M$. For example, in the case of $Q_{1} Q_{5}=2, M$ is the Eguchi-Hanson space. When the FI parameters go to zero, it reduces to the singular space, $C^{2} / Z_{2}$. Similarly, we can show that when the FI terms go to zero, $M$ defined by the equations (3.12) and (3.13) becomes the singular space (appendix A)

$$
M_{0}=C^{2} \times \frac{C^{2}}{w} \times \frac{C^{2}}{w^{2}} \times \cdots \frac{C^{2}}{w^{N-1}} .
$$

The singularity of $M_{0}$ corresponds to the cycle of length $N$ of the permutation group $S_{N}$. Associated with this singularity is a chiral primary operator with dimension $(h, \bar{h})=$ $\left(\frac{N-1}{2}, \frac{N-1}{2}\right)$. This result has appeared before in $\|$ using a different approach. In order to recover the singularity structure corresponding to all the other cycles and the corresponding chiral primaries, we may have to consider D-terms (similar to equations (3.12) (3.13) with FI parameters equal to zero ) corresponding to effective $U(1)$ theories describing the splitting processes 21] $\left(Q_{1}, Q_{5}\right) \rightarrow\left(Q_{1}^{\prime}, Q_{5}^{\prime}\right)+\left(Q_{1}^{\prime \prime}, Q_{5}^{\prime \prime}\right)$.

We would like to mention that all the chiral primaries have been obtained using the symmetric product description of the instanton moduli space on $T^{4}$. 3]

\subsection{The Bound State in the Higgs Phase}

Having discussed the moduli space that characterizes the SUSY ground state we can discuss the fluctuations of the transverse vector multiplet scalars $X_{m}$ and $Y_{m}, m=1,2,3,4$. In the Higgs phase since $\left\langle X_{m}\right\rangle=\left\langle Y_{m}\right\rangle=0$ and $\chi=\bar{\chi}$ lies on the surface defined by Eqn. (3.9). The relevant action of fluctuations in the path integral is,

$$
S=\sum_{m} \int d t d x_{5}\left(\operatorname{tr}_{U\left(Q_{5}\right)} \partial_{\alpha} X_{m} \partial^{\alpha} X_{m}+t r_{U\left(Q_{1}\right)} \partial_{\alpha} Y_{m} \partial^{\alpha} Y_{m}\right)+\int d t d x_{5}\left(V_{2}+V_{3}\right)
$$

We restrict the discussion to the case when $Q_{5}=1$ and $Q_{1}$ is arbitrary. In this case the matrix $X_{m}$ is a real number which we denote by $x_{m}$. 
$\chi$ is a complex column vector with components $\left(A_{a^{\prime}}, B_{a^{\prime}}\right), a^{\prime}=1, \ldots, Q_{1}$. Since we are looking at the fluctuations of the $Y_{m}$ only to quadratic order in the path integral, the integrals over the different $Y_{m}$ decouple from each other and we can treat each of them separately. Let us discuss the fluctuation $Y_{1}$ and set $\left(Y_{1}\right)_{a^{\prime} b^{\prime}}=\delta_{a^{\prime} b^{\prime}} y_{1 a^{\prime}}$. Then the potential $V_{3}$, (3.5) becomes

$$
V_{3}=\sum_{a^{\prime}}\left(\left|A_{a^{\prime}}\right|^{2}+\left|B_{a^{\prime}}\right|^{2}\right)\left(y_{1 a^{\prime}}-x_{1}\right)^{2}
$$

We will prove that $\left|A_{a^{\prime}}\right|^{2}+\left|B_{a^{\prime}}\right|^{2}$ can never vanish if the FI terms are non-zero. In order to do this let us analyze the complex D-term equation (3.9)

$$
A_{a^{\prime}} B_{b^{\prime}}+\left[N_{1}^{(1)}, N_{2}^{(1) \dagger}\right]_{a^{\prime} b^{\prime}}=\frac{\zeta_{c}}{Q_{1}} \delta_{a^{\prime} b^{\prime}}
$$

We can use the complex gauge group $G L\left(C, Q_{1}\right)$ to diagonalize the complex matrix $N_{1}^{(1)}$ [31. Then, the above equation becomes

$$
A_{a^{\prime}} B_{b^{\prime}}+\left(n_{a^{\prime}}-n_{b^{\prime}}\right)\left(N_{2}^{(1) \dagger}\right)_{a^{\prime} b^{\prime}}=\frac{\zeta_{c}}{Q_{1}} \delta_{a^{\prime} b^{\prime}}
$$

For $a^{\prime} \neq b^{\prime}$, this determines the non-diagonal components of $N_{2}^{(1)}$

$$
\left(N_{2}^{(1) \dagger}\right)_{a^{\prime} b^{\prime}}=-\frac{A_{a^{\prime}} B_{b^{\prime}}}{n_{a^{\prime}}-n_{b^{\prime}}}
$$

For $a=b$, we get the equations

$$
A_{a^{\prime}} B_{a^{\prime}}=\frac{\zeta_{c}}{Q_{1}}, a^{\prime}=1, . ., Q_{1}
$$

which imply that

$$
\left|A_{a^{\prime}}\right|\left|B_{a^{\prime}}\right|=\frac{\left|\zeta_{c}\right|}{Q_{1}}
$$

with the consequence that $\left|A_{a^{\prime}}\right|$ and $\left|B_{a^{\prime}}\right|$ are non-zero for all $a^{\prime}=1, . ., Q_{1}$. This implies that $\left.\left(\left|A_{a^{\prime}}\right|^{2}+\left|B_{a^{\prime}}\right|^{2}\right)>0\right)$, and hence the fluctuation $\left(y_{1 a^{\prime}}-x_{1}\right)$ is massive. If we change variables $y_{1 a^{\prime}} \rightarrow y_{1 a^{\prime}}+x_{1}$, then $x_{1}$ is the only flat direction. This corresponds to the global translation of the 5 -brane in the $x_{1}$ direction.

A similar analysis can be done for all the remaining directions $m=2,3$, 4 with identical conclusions. This shows that a non-zero FI term implies a true bound state of the $Q_{5}=1$, $Q_{1}=N$ system. This result was previously presented in [5]. If $F I=0$, then there is no 
such guarantee and the system can easily fragment, due to the presence of flat directions in $\left(Y_{m}\right)_{a^{\prime} b^{\prime}}$

What the above result says is that when the FI parameters are non-zero the zero mode of the fields $\left(Y_{m}\right)_{a^{\prime} b^{\prime}}$ is massive. If we regard the zero mode as a collective coordinate then the Hamiltonian of the zero mode has a quadratic potential which agrees with the near horizon limit of the Liouville potential derived in section 2.3.

The general case with an arbitrary number of $Q_{1}$ and $Q_{5}$ branes seems significantly harder to prove but we are optimistic.

\section{SCFT on the Moduli Space of Non-Commutative Gauge Theory}

Here we briefly explore the possible connection of this work with Non-commutative geometry. As has been discussed by several authors, the presence of a non-zero $B_{N S}$ in the near horizon geometry of a D-brane system wrapped on a torus, will modify the boundary conditions of the open strings along the brane directions. Then, the brane world volume Yang-Mills theory is substituted by a Yang-Mills theory on the non-commutative torus.

In the D1/D5 system, the configurations which break one half of the supersymmetries of the 5-brane theory are self-dual connections of this NCYM theory. The moduli space of these connections with the $B_{N S}$ turned on, will then define a conformally invariant sigma-model.

The $N=4$ symmetric product SCFT with $c=6 Q_{1} Q_{5}$ has been constructed in some detail and its 20 dim. moduli space can be explicitly constructed in terms of operators of the SCFT [3]. Of the four operators corresponding to the blowup modes of the orbifold

singularity, 3 correspond to $B_{i j}^{(+)}$, the self dual part of the $B_{i j}$ in the NS sector of the closed string theory and the remaining one corresponds to the $\theta$ parameter.

We wish to propose that this new sigma-model, coming from the noncommutative YangMills theory, is at some point in the moduli space of the symmetric product SCFT, deformed by the addition of the four marginal deformations corresponding to the moduli $B_{i j}^{+}$and $a C_{0}+b C_{4}$.

After this work was completed we became aware of [32 which has some overlap with this work. 


\section{Acknowledgement}

We would like to acknowledge Justin David for collaboration during the early stages of this work, and his critical comments on the manuscript. We would like to thank Fawad Hassan for a very helpful correspondence regarding [25]. We (SRW,KPY) would also like to thank the 'Extended Workshop in String Theory', at the Mehta Research Institute, for hospitality during the final stages of this work. We thank Ashoke Sen and David Tong for useful comments on some aspects of this work related to the moduli space of instantons.

\section{A Derivation of (3.10)}

We now study the limiting case of $M=T^{*} C P^{Q_{1} Q_{5}-1}$ as $\zeta \rightarrow 0$. However this seems presently hard to do and hence we set $\zeta=0$ in Eqn. (3.9), and explicitly demonstrate that these equations describe the symmetric product of $\left(Q_{1} Q_{5}-1\right)$ copies of $C^{2}=C \times C$ where $C$ denotes the complex plane.

In the following we will, for convenience, use the symbol $N$ for $Q_{1} Q_{5}$.

Since we are dealing with $N U(1)$ hyper multiplets, it is convenient to denote the hypermultiplets by $A_{a}, B_{a}^{+}, a=1, \cdots, N$. Then Eqns. (3.9) become (we have set $\zeta=0$ ),

$$
\begin{gathered}
\sum_{a=1}^{N}\left(\left|A_{a}\right|^{2}-\left|B_{a}\right|^{2}\right)=0 \\
\sum_{a=1}^{N} A_{a} B_{a}=0
\end{gathered}
$$

The hypermultiplets constitute $2 N$ complex, or equivalently $4 N$ real variables. These variables are not invariant under the $U(1)$ ( $A$ has $U(1)$ charge 1 and $B$ has charge -1$)$. Using (A.1a) and dividing by $U(1)$ leaves us with $4 N-2$ real, or $2 N-1$ complex variables which can be parametrized by the $2(N-1)+N=3 N-2$ (complex) gauge invariant variables

$$
\begin{aligned}
P_{a} & =A_{a} B_{a}, \quad a=1, \cdots, N \\
M_{a} & =A_{a} B_{N}, \quad a=1, \cdots, N-1 \\
N_{a} & =B_{a} A_{N}, \quad a=1, \cdots, N-1
\end{aligned}
$$

subject to the $N-1$ obvious conditions

$$
P_{a} P_{N}=M_{a} N_{a}, \quad a=1, \cdots, N-1
$$


Let us now use the complex equation (A.1b), which translates to

$$
\sum_{a}^{N} P_{a}=0
$$

The $N$ constraints involving the $3 N-2$ variables $P, M, N$ can be solved in terms of $(N-1)$ pairs of complex numbers $\left(\eta_{i}, \xi_{i}\right), i=1, \cdots,(N-1)$

$$
\begin{aligned}
P_{a} & =w^{a} P_{N}, \quad w^{N}=1, \quad P_{N}=\prod_{i=1}^{N-1}\left(\eta_{i} \xi_{i}\right)^{1 / 2} \\
M_{a} & =\sqrt{w_{N}^{a}} \eta_{a} \prod_{j \neq a}^{N-1}\left(\eta_{j} \xi_{j}\right)^{1 / 2} \\
N_{a} & =\sqrt{w_{N}^{a}} \xi_{a} \prod_{j \neq a}^{N-1}\left(\eta_{j} \xi_{j}\right)^{1 / 2}
\end{aligned}
$$

This solution is characterized by a point in $(N-1)$ copies of $C^{2}$. However there are identifications which can be characterized in terms of the cycles of the symmetric group $S_{N}$. For example consider the action of the largest cycle corresponding to the diagonal action $\left(\xi_{a}, \eta_{a}\right) \rightarrow e^{i 2 \pi a / N}\left(\xi_{a}, \eta_{a}\right) \equiv w^{a}\left(\xi_{a}, \eta_{a}\right)$ where $w^{N}=1$.

It is clear that such a transformation leaves the gauge invariant coordinates $P_{a}, M_{a}$ and $N_{a}$ invariant. Hence the $(N-1)$ copies of $C^{2}$ have to be quotiented as follows:

$$
C^{2} \times \frac{C^{2}}{w} \times \frac{C^{2}}{w^{2}} \times \cdots \frac{C^{2}}{w^{N-1}}
$$

\section{References}

[1] A. Strominger and C. Vafa, "Microscopic Origin Of The Bekenstein-Hawking Entropy," Phys. Lett. 379 (1996) 99, hep-th/9601029

[2] J.R.David, G.Mandal and S.R.Wadia, "Absorption and Hawking radiation of minimal and fixed scalars, and AdS/CFT correspondence", Nucl.Phys. B544 (1999) 590, hepth/9808168

[3] J.R.David, G.Mandal and S.R. Wadia, "D1/D5 moduli in SCFT and gauge theory and Hawking radiation", hep-th/9907075

[4] N. Seiberg and E.Witten, "The D1/D5 system and singular CFT", hep-th/9903224 
[5] S.R. Wadia, Lectures given at the "Advanced School on Supersymmetries in the Theories of Fields, Strings and Branes, Santiago de Compostela, Spain, July 26-31, 1999 (available from http://www-fp.usc.es/ theory)

[6] C.G. Callan and J. Maldacena, "D-brane Approach to Black Hole Quantum Mechanics", Nucl. Phys. B472 (1996) 591, hep-th/9602043

[7] S.F Hassan and S.R. Wadia, "Gauge Theory Description of D-brane Black Holes: Emergence of the Effective SCFT and Hawking Radiation", Nucl. Phys.B526(1998) 311, hep-th/971213

[8] N. Seiberg and E. Witten, talks given at Strings99

[9] N. Seiberg and E.Witten, "Noncommutative Geometry and String theory",JHEP 9909:032,1999, hep-th/9908142

[10] A. Connes, M. Douglas, A.Schwarz, "Non-commutative Geometry and Matrix Theory: Compactification on Tori", JHEP 9802 (1998) 003, hep-th/9711162

[11] M. Douglas and C. Hull, " D-Branes and the non-commutative torus", JHEP9802:008(1998), hep-th/9711165

[12] V. Schomerus, "D-Branes and deformation quantization", JHEP-9906:030(1999), hepth/9903205

[13] F. Ardalan, H. Arfaei and M.M. Sheikh-Jabbari, " Non- commutative geometry from strings and branes", JHEP-02,016 (1999), hep-th/9810072

[14] Y-K.E Cheung and M. Krogh, " Non-commutative geometry from 0-branes in a background B-field", Nucl. Phys. B528 (1998) 185, hep-th/9803031

[15] C.-S. Chu and P.-M. Ho, " Non-commutative open strings and D-Brane", Nucl. Phys. B550 (1999) 151, hep-th/9812219

[16] A. Hashimoto and N. Itzhaki, " Non-commutative Yang-Mills and the Ads/CFT correspondence", hep-th/9907166

[17] D. Bigatti and L. Susskind, " Magnetic Fields, Branes and Non-commutative geometry", hep-th/9908056 
[18] J. M. Maldacena, talk given at Strings99

[19] J.M Maldacena and J.G. Russo, "The large-N limit of Non-commutative gauge theories", hep-th/9908134

[20] N. Nekrasov and A. Schwarz," Instantons on noncommutative $R^{4}$ and $(2,0)$ superconformal six dimensional theory", Commun. Math. Phys. 198 (1998) 689, hepth/9802068

[21] J.R David, PhD. Thesis (1999) (to appear on hep-th)

[22] J.P. Gauntlett, "Intersecting Branes", hep-th/9705011

[23] See, e.g., A. A. Tseytlin, "Harmonic Superpositions of M-Branes", Nucl.Phys. B475 (1996) 149-163, hep-th/9604035

[24] J. C. Breckenridge, G. Michaud, R. C. Myers, "More D-brane bound states", Phys.Rev. D55 (1997) 6438-6446, hep-th/9611174

[25] S.F.Hassan, "T-Duality, Space-time Spinors and R-R fields in curved backgrounds", hep-th 9907152

[26] Juan M. Maldacena, "The Large N Limit of Superconformal Field Theories and Supergravity", Adv.Theor.Math.Phys. 2 (1998) 231-252, hep-th/9711200

[27] For a review of mass-formulae of supergravity solutions preserving various amount of supersymmetry, see, e.g., N.A Obers and B. Pioline, "U-duality and M-theory", hep-th/9809039

[28] C. Vafa, "Gas of D-branes and Hagedorn Density of BPS States", hep-th/9511088

[29] R. Dijkgraaf, "Instanton Strings and Hyper-Kahler Geometry", Nucl. Phys. B543 (1999) 545, hep-th/9810210

[30] S.Ferrara and R.E. Kallosh, "Supersymmetry and Attractors", Phys. Rev D54 1514$1524,1996$.

[31] E. Witten, "On the Conformal field theory of the Higgs branch",JHEP 9707:003,1997, hep-th/9707093 
[32] A. Mikhailov, "D1/D5 System and Noncommutative Geometry", hep-th/9910126 